\title{
Erratum to: Agonist anti-GITR monoclonal antibody and stereotactic radiation induce immune-mediated survival advantage in murine intracranial glioma
}

Mira A. Patel ${ }^{1+}$, Jennifer E. Kim ${ }^{1 \dagger}$, Debebe Theodros ${ }^{1}$, Ada Tam² ${ }^{2}$ Esteban Velarde ${ }^{3}$, Christina M. Kochel², Brian Francica ${ }^{2}$, Thomas R. Nirschl ${ }^{2}$, Ali Ghasemzadeh², Dimitrios Mathios ${ }^{4}$, Sarah Harris-Bookman ${ }^{4}$, Christopher C. Jackson ${ }^{4}$, Christina Jackson ${ }^{4}$, Xiaobu Ye ${ }^{4}$, Phuoc T. Tran ${ }^{2,3,6}$, Betty Tyler ${ }^{4}$, Vladimir Coric ${ }^{5}$, Mark Selby ${ }^{5}$, Henry Brem ${ }^{1,4}$, Charles G. Drake ${ }^{6}$, Drew M. Pardoll ${ }^{2}$ and Michael Lim ${ }^{1,4^{*}}$

\section{Erratum}

Unfortunately, after publication of this article [1], it was noticed that a funding source was not mentioned. Bristol-Myers Squibb was intended to be included in the 'Financial Support' section of the article.

\footnotetext{
Author details

${ }^{1}$ The Johns Hopkins University School of Medicine, Baltimore, USA.

${ }^{2}$ Department of Oncology, Baltimore, USA. ${ }^{3}$ Department Radiation Oncology, Baltimore, USA. ${ }^{4}$ Department of Neurosurgery, The Johns Hopkins University School of Medicine, 600 N. Wolfe St. Phipps Building Rm 123, Baltimore 21287, MD, USA. ${ }^{5}$ Bristol-Myers Squibb Company, San Francisco, CA, USA.

${ }^{6}$ The Brady Urological Institute, Baltimore, USA.
}

Received: 24 October 2016 Accepted: 25 October 2016 Published online: 04 November 2016

\section{Reference}

1. Patel MA, Kim JE, Theodros D, Tam A, Velarde E, Kochel CM, Francica B,

Nirschl TR, Ghasemzadeh A, Mathios D, Harris-Bookman S, Jackson CC, Jackson C, Ye X, Tran PT, Tyler B, Coric V, Selby M, Brem H, Drake CG,

Pardoll DM, Lim M. Agonist anti-GITR monoclonal antibody and stereotactic radiation induce immune-mediated survival advantage in murine intracranial glioma. J Immunothera Cancer. 2016;4:28. doi:10.1186/s40425-016-0132-2.

\footnotetext{
* Correspondence: mlim3@jhmi.edu

${ }^{\dagger}$ Equal contributors

'The Johns Hopkins University School of Medicine, Baltimore, USA ${ }^{4}$ Department of Neurosurgery, The Johns Hopkins University School of Medicine, 600 N. Wolfe St. Phipps Building Rm 123, Baltimore 21287, MD, USA
} 